\title{
KYSTE EPIDERMOIDE INTRACRANIEN
}

\author{
K. SOMAI , L. HARZALLAH, I. HAMDI, L. BEN CHERIFA, S. KRIAA, \\ H. AMARA, D. BAKIR, C. KRAIM \\ SERVICE D'IMAGERIE MÉDICALE FARHAT HACHED. \\ RUE IBN EL JAZZAR 4000 SOUSSE
}

\begin{abstract}
But: illustrer l'apport de l'Imagerie par résonance magnétique (IRM) dans le diagnostic de 2 cas de kystes épidermoïdes. Matériels: 2 cas de kystes épidermoïdes prouvés histologiquement ont été étudiés. Tous les patients ont été examinés avec une IRM conventionnelle (SET1, SET2, FLAIR) et par imagerie de diffusion.

Résultat: les lésions étaient localisées dans l'angle ponto-cérébelleux droit et la fosse temporale droite. Toutes les lésions étaient hyperintenses en T2, hypointenses en T1 et très hyperintenses en Diffusion.

Conclusion: Les séquences FLAIR et surtout de Diffusion sont les séquences les plus utiles pour le diagnostic du kyste épidermoïde.

Mots clés: Kyste épidermoïde - IRM
\end{abstract}

Introduction: Aim : To evaluate the value of magnetic resonance imaging (MRI) in the diagnosis of 2 cases of epidermoid cysts.

Materieal and Methods: 2 cases of histopathologically proven epidermoid cysts were studied. All patients were examined with conventional MRI (T1WI, T2WI, FLAIR) and Diffusion-weighted imaging (DWI).

Results: the lesions were located in the right cerebellopontine angle and the right temporal fossa. All lesions were hyperintense on T2, hypointense on T1and strikingly hyperintense on DW.

Conclusion: New MR techniques such as FLAIR and DWI are most helpful tools in the specific preoperative diagnosis which is important for surgical guidance.

\section{INTRODUCTION}

Le kyste épidermoïde est une lésion congénitale dysgénétique rare de l'homme.

L'imagerie en coupe, essentiellement I'IRM, est capitale pour le diagnostic positif. En effet cette lésion présente un aspect pratiquement pathognomonique.

Nous rapportons deux cas rares d'un kyste épidermoïde de l'angle pontocérébelleux droit et de la fosse temporale droite, en précisant l'apport de la séquence de diffusion dans le diagnostic positif et différentiel.

\section{OBSERVATION 1}

II s'agit d'un homme âgé de 32 ans, sans antécédents pathologiques notables qui consulte pour des céphalées. L'examen clinique est sans particularités notamment l'examen ORL. L'IRM trouve une lésion kystique extra axiale de l'APC droit de $4 \mathrm{~cm}$ de grand axe à contours polyédriques irréguliers exerçant un effet de masse sur le parenchyme cérébelleux en regard et englobant en partie les structures nerveuses homolatérales. Elle est en hypo signal $T 1$, en hyper T2 non atténué sur la séquence FLAIR et non rehaussée après injection de Gadolinium. Cette tumeur présente un hyper signal franc sur la séquence de diffusion avec un ADC diminué par rapport au LCR. Cet aspect est pathognomonique du kyste épidermoide.
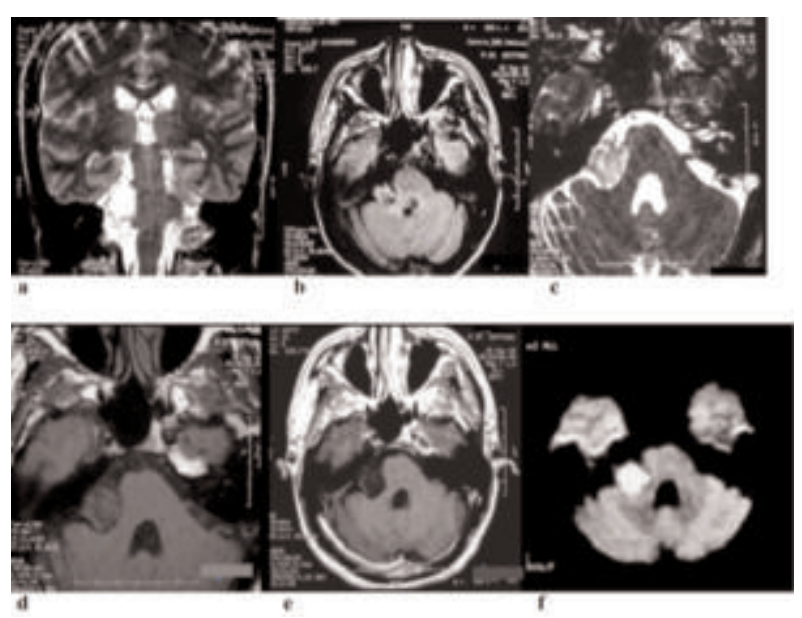

Fig. 1 : Kyste épidermoide de I'APC droit :

(a) hyper signal T2 (b) non atténué sur la séquence FLAIR, (c) hyper signal modéré hétérogène en FIESTA, (d) hypo signal T1, (e) non rehaussée après injection de Gadolinium, (f) avec hyper signal franc sur la séquence de diffusion. 


\section{OBSERVATION 2}

II s'agit d'un patient de 20 ans, sans antécédents pathologiques notables qui consulte pour otalgie droite sans fièvre. L'examen ORL est sans anomalies avec un tympan d'aspect morphologique normal et absence de troubles audiométriques. Le scanner trouve un processus expansif intracrânien sus- tentoriel extra axial de la fosse temporale droite de $5 \mathrm{~cm}$ de grand axe. Cette tumeur exerce un effet de masse modéré sur le parenchyme cérébral adjacent avec une importante ostéolyse de la voûte crânienne en regard et des cellules mastoïdiennes homolatérales avec lyse du tegmen tympani et pneumocephalie.

L'IRM retrouve la lésion en hypo signal T1 (fig.2c), en hyper T2 (fig2a) non atténué sur la séquence FLAIR (fig.2b) non rehaussée après injection de Gadolinium (fig.2d) avec un hyper signal franc sur la séquence de diffusion et un ADC diminué par rapport au LCR. II s'agit d'un kyste épidermoide de l'écaille de l'os temporal droit confirmé par l'examen anatomopathologique.
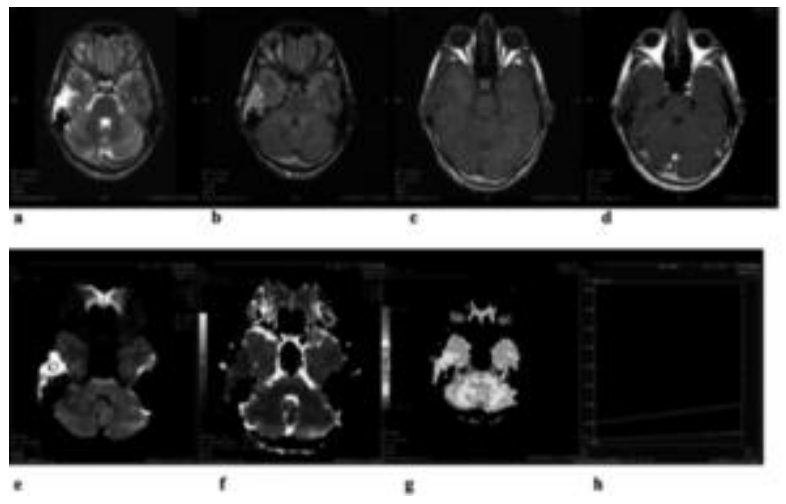

Fig. 2 : Kyste epidermoide de la fosse temporale droite. La lésion est en hypersignal T2 (a),non atténuée sur la séquence FLAIR (b),elle est en hyposignal T1(c) et en hypersignal franc en diffusion (e) .

La cartographie d'ADC en échelle de gris (f): LCR en blanc (ADC élevé); kyste en gris foncé (ADC bien inférieur a celui du LCR).

La cartographie d'ADC en couleur (g): ADC du kyste en bleu clair, inférieur à celui du LCR en rouge.

Courbe ( $h)$ : $A D C$ en rose de la lésion, en vert du parenchyme cérébral.

\section{DISCUSSION}

Le kyste épidermoide (KE) encore appelé tumeur perlée est une lésion congénitale dysembryoplasique bénigne due à l'inclusion anormale d'éléments épithéliaux dans le tube neural lors de sa fermeture entre la 3éme et la 5éme semaine de vie intratérine ou plus tardivement lors de la formation des vésicules cérébrales ou de l'enfouissement des placodes auditives (1).

Elle peut être secondaire aussi à l'introduction de fragments épidermiques dans les éspaces sous arachnoidiens après un traumatisme ou un acte chirurgical $(2,3)$. La croissance de cette tumeur se fait lentement par desquamation des couches épidermiques entraînant une accumulation de kératine et d'autres produits de dégra- dation formant ainsi un processus mou qui se moule et englobe les structures adjacentes (1).

Le KE représente 0.2 à $1 \%$ de l'ensemble des tumeurs intracrâniennes primitives et moins de $5 \%$ des tumeurs de l'angle ponto-cérébelleux $(4,5,6)$. Cette lésion se révèle à tous les âges et sans préférence de sexe ; cependant un pic de fréquence s'observe entre la 3éme et 5éme décade $(1,7)$

Sur le plan clinique, les kystes épidermoïdes ont une symptomatologie d'emprunt, des symptômes auditifs ou vestibulaires en cas d'irritation du paquet acousticovestibulaire ou des troubles sensitifs d'une hémiface par compression du nerf trijumeau. Ailleurs, des céphalées banales peuvent révéler la lésion. La découverte fortuite est également possible.

Ses localisations les plus fréquentes sont l'angle pontocérébelleux (3éme après le méningiome et le schwannome), la fosse crânienne moyenne, la région suprasellaire et la région quadrigéminale (7). Les localisations au sein du V4 sont exceptionnelles.

Macrospiquement, le KE se présente sous la forme d'une masse bien limitée de couleur blanche nacrée, avasculaire avec une surface irrégulière et nodulaire, entouré d'une fine capsule. Son contenu, dont la consistance évoque la cire de bougie est composé de lamelles blanchâtres $(6,8,9)$.

Microscopiquement, sa paroi est formée d'une mince capsule de tissus conjonctifs sur laquelle repose un épithélium kératinisé monostratifié interne. Son contenu est formé d'un matériel riche en cholestérol provenant de la dégradation de la kératine $(4,9,10)$.

La TDM montre une masse aux contours irréguliers, lobulés, souvent hétérogène, hypodense, exceptionnellement hyperdense $(7,11)$. Les calcifications sont très rares[8,9], se voient dans 10 à $25 \%$ (12) et siégent en péricapsulaire. Après injection de produit de contraste, le $\mathrm{KE}$ ne se rehausse pas. Exceptionnellement on peut noter une prise de contraste périphérique liée au rehaussement d'une structure péritumorale.

En IRM, le signal du KE est celui du liquide cérébro-spinal (LCS) en hyposignal T1 et en hypersignal T2, mais le signal est souvent hétérogène notamment en T2. En densité de proton, le signal est hétérogène et légèrement supérieur à celui du LCS et on observe occasionnellement un anneau en hypersignal autour du KE par piégeage du LCS $(13,14,15)$.

D’autres séquences, facilitent grandement le diagnostic et précisent mieux l'extension et l'éventuelle existence de reliquat post opératoire : il s'agit des séquences FLAIR T2, diffusion et CISS ou 3D écho de spin rapide (11).

L'imagerie de diffusion qui permet en quelques secondes une mesure des mouvements microscopiques des molécules d'eau est une séquence clé. En effet, le KE apparaît en hypersignal avec un ADC inférieur à celui observé dans les espaces liquidiens, voisin de celui du parenchyme cérébral. L'hyper signal en diffusion s'explique principalement par l'important allongement de T2 (T2 shine through effect) $(16,18,19)$.

Le problème de diagnostic différentiel se pose essentiellement avec le kyste arachnoïdien (KA) ou plus 
rarement avec le kyste neurentérique.

Cependant,certains arguments comme l'hétérogénéité du signal en T1, les contours nets irréguliers en « carte géographique ", ainsi que l'extension dans les espaces sous arachnoïdiens plaident en faveur du KE. En plus, deux séquences ont un apport important: La séquence FLAIR, qui supprime le signal du LCS, permet de différencier entre kyste épidermoïde et kyste arachnoïdien. Avec cette séquence, le KE garde un signal élevé, alors que le $K A$ voit son signal supprimé. De même pour la séquence Diffusion, le KE est en hypersignal alors que le KA est en hyposignal $(12,15)$.

\begin{tabular}{|c|c|c|c|c|}
\hline \multirow[b]{2}{*}{ Lènian } & \multicolumn{4}{|c|}{ Apparenes var les differentes séquences } \\
\hline & TI & 72 & Fair & Diffusion \\
\hline $\begin{array}{l}\text { Kyste } \\
\text { Fipidermoide }\end{array}$ & byprintense & boperintens & vignal alowe & bpenviped \\
\hline $\begin{array}{l}\text { Kysle } \\
\text { Aracheoidien }\end{array}$ & hypenatimes & loperiaiane: & hyporignal & hyposignal \\
\hline
\end{tabular}

Tableau I. : Diagnostic différentiel entre kyste épidermoïde et kyste arachnoidien. Caractéristiques à L'IRM

Le traitement des KE est chirurgical. II consiste à vider le contenu kystique et à pratiquer une exérèse complète de la paroi. Dans le cadre de la surveillance postopératoire, l'imagerie de diffusion permet d'établir le caractère complet ou non de l'exérèse (20). En cas de résidu tumoral, une surveillance annuelle permet d'évaluer le potentiel évolutif du résidu.

\section{CONCLUSION}

Le kyste épidermoide est une tumeur congénitale rare d'évolution lente découverte à tout âge.

Sur les séquences conventionnelles, les kystes épidermoides apparaissent iso intenses ou discrètement hyper intenses par rapport au LCR. En imagerie de diffusion, les kystes épidermoïdes sont très hyper intenses et se distinguent très facilement du LCR et des kystes arachnoïdiens qui apparaissent hypo intenses.

\section{REFERENCES}

[1] Sarrazin J-L, Marsot-Dupuch K, Chayas A. Pathologie de l'angle ponto-cerebelleux. J Radiol $2006 ; 87: 1765-82$.

[2] Smith Cml, Timperley WR. Multiple intraspinal and intracranial epidermoids and lipomata following gunshot injury. Neuropathol Appl Neurobiol 1984;10: 235239.

[3] Vinchon M, Lejeune JP, Krivosic I. Les kystes dermoides et epidermoides cranio-encephaliques : classification et pathogenie. Neurochirurgie $1995 ; 41$ 29-37. [4] Bensaid A, Navarro C, Cejas C, Arhan JM, Gangi A, Dietemann JL. Aspect TDM et IRM des kystes epidermoides et dermoides intracraniens. Feuillets de radiologie $1994 ; 34: 55-65$

[5] Chagnon S, Vallee C, Blery M, Chevrot A. kyste epidermoide. Encycl Med Chir (Paris-France), Radiodiagnostic. Neuroradiologie-Appareil Locomoteur, 31490 B10, 1992: 4 p.

[6] Dillon WP, Som PM , Fullterton GD. Hypointense MR signal in chronically inspissated sinonasal secretions. Radiology $1990 ; 174: 73-78$.

[7] Megdiche Bazarbacha H, Nagi S, Douira W, Sebai R, Belghith S, Touibi S. Jneuroradiol 2004; 31, 150-152.

[8] Cosnard G, Galidie G, Cordoliani YS, Legall R. Tumeurs rares de l'angle ponto-cerebelleux. Ann de Radiologie 1992;35:36-43.

[9] Nagashima C, Takahama M, Sakaguchi A. Dense cerebellopontine epidermoid cyst. Surg Neurol 1982; 17: 172-177.

[10] Doll A, Abu Eid M, Kehrli P, Esposito P, Gillis C, Bogorin A, Jaques C, Dietemann JL. Aspects en sequences Flair, CISS-3D et en imagerie de diffusion des kystes epidermoides intracraniens. J Neuroradiol $2000 ; 27: 101-106$.

[11] Sarrazin JL, Helie O, Cordoliani YS. Tumeurs de l'angle ponto-cerebelleux chez l'adulte. J Radiologie $2000 ; 81: 675-690$.

[12] Osborn AG, Preece MT. Intracranial cysts: Radiologic-Pathologic correlation and Imaging Approach. Radiology 2006; 239: 650-664.

[13] Gao P, Osborn AG, Smirniotopoulos JG. Epidermoid tumor of the cerebellopontine angle. AJNR 1992; 13: 863-872.

[14] Kallmes DF, Provenzale JM, Cloft HJ, Clendon RE. Typical and atypical MR imaging features of intracranial epidermoid tumors. AJR 1997; 169:883-887.

[15] Bonneville F, Sarrazin JL, Marsot-Dupuch K, Iffenecker C, Cordoliani YS, Doyon D, Bonneville JF. Unusual lesions of the cerebellopontine angle: A segmental approach. Radiographics 2001; 21: 419-438.

[16] Oppenheim C, Naggara O, Rodrigo S, Bienvenu M, Meder JF, Hamon M, Gauvrit $\mathrm{Y}$, Menegon $\mathrm{P}$, Cosnard $\mathrm{G}$. Imagerie par résonance magnétique de diffusion de l'encéphale chez l'adulte : technique, résultats normaux et pathologiques. Encycl Med Chir ( Elsevier, Paris),Radiodiagnostic I-II- Squelette normalNeuroradiologie-Appareil locomoteur, 30-806-c-10, 2005, 17p

[17] Rao VJ, James RA, Mitra D Imaging characteristics of common suprasellar lesions with emphasis on MRI findings. Clinical Radiology 2008; 63 : 939-947. [18] Chen S, Ikawa F, Kurisu K, Arita K, Takaba J, Kanou Y.Quantitative MR evaluation of intracranial epidermoid tumors by fast fluid-attenuated inversion recovery imaging and echoplanar diffusion-weighted imaging. Am J Neuroradiol 2001;22:1089-96.

[19] Annet L, Duprez T, Grandin C, Dooms G, Collard A, Cosnard G.Apparent diffusion coefficient measurements within intracranial epidermoidcysts in six patients. Neuroradiology 2002:44:326-8.

[20] Dechambre S, Duprez T, Lecouvet F, Raftopoulos C, Cosnard G

Diffusion-weighted MRI postoperative assessment of an epidermoid tumour in the cerebellopontine angle. Neuroradiology 1999; 41:829-831. 